\title{
An experimental study of hiss-triggered chorus emissions at low latitude
}

\author{
Rajesh Singh ${ }^{1}$, R. P. Patel ${ }^{1}$, R. P. Singh ${ }^{1}$, and Lalmani $^{2}$ \\ ${ }^{1}$ Atmospheric Research Laboratory, Physics Department, Banaras Hindu University, Varanasi-221005, India \\ ${ }^{2}$ Department of Physics, Regional Engineering College, Srinagar, Camp Classes, Jammu-180001, India
}

(Received December 21, 1998; Revised August 20, 1999; Accepted September 20, 1999)

\begin{abstract}
Hiss-triggered chorus emissions recorded at low-latitude ground station Gulmarg (geomag. lat., $24^{\circ} 26 \mathrm{~N}$; geomag. long., $147^{\circ} 09 \mathrm{E}, L=1.28$ ) on March 8,1986 are reported. From the detailed spectral analysis of these emissions, it is found that the chorus is hiss-triggered and each chorus element has tendency to originate from the hiss band. Based on the theory of Helliwell, and previous satellite VLF data, a possible generation mechanism of low-latitude hiss-triggered chorus emissions is proposed. An attempt has been made to find out the chorus source location and the length of resonance region from which the various parameters associated with generation mechanism are determined.
\end{abstract}

\section{Introduction}

Magnetospheric VLF/ELF emissions are classified into two different types namely: (1) unstructured hiss and (2) structured and discrete chorus (Helliwell, 1965; Sazhin, 1982; Hayakawa et al., 1990; Sazhin and Hayakawa, 1992; Hattori and Hayakawa, 1994). Various features of these emissions have been studied from time to time. However, fundamental problems,including whether these two types of emissions are essentially different or not and the link between the two, are still unsolved, and further investigation is required. The study of the hiss triggered chorus emissions play significantly important role in understanding of the above mentioned problems.

Unlike mid and high latitude emissions, low latitude VLF emissions have not been used much for exploring the low latitude inner magnetosphere. The main reason being the fact that the propagation characteristics of VLF emissions in the low latitude ionosphere are not properly known. An understanding of the generation mechanism of these VLF emissions observed at low latitudes would be most useful for inferring the properties of high energy trapped electrons. During the course of our analysis of the huge amount of VLF data collected during January-July, 1986 at Gulmarg ( $L=1.28$; geomag. lat., $24^{\circ} 26 \mathrm{~N}$; geomag. long., $147^{\circ} 09 \mathrm{E}$ ), we have found some excellent records of hiss-triggered chorus emissions which we reproduce here with a discussion of their most probable generation mechanism. In order to clarify the fundamental problems of whether two types of VLF emissions are essentially same or different and to infer the properties of the high energy trapped electrons, the study of hiss-triggered chorus emissions recorded at our low latitude ground station Gulmarg is of the great importance.

The experimental observations made so far on these emissions strongly support the suggestions made earlier that these

Copy right $(\mathrm{C})$ The Society of Geomagnetism and Earth, Planetary and Space Sciences (SGEPSS); The Seismological Society of Japan; The Volcanological Society of Japan; The Geodetic Society of Japan; The Japanese Society for Planetary Sciences. emissions are produced in the equatorial zone of the magnetosphere by trapped energetic electrons. Out of various mechanisms proposed from time to time, the transverse resonance interaction between whistler mode waves and energetic electrons could explain most of the characteristics of VLF emissions recorded on the ground stations (Helliwell, 1967). The generation mechanism of spontaneous chorus emissions has been studied by several workers (Burton and Holtzer, 1974; Goldstein and Tsurutani, 1984; Hayakawa et al., 1984; Hattori and Hayakawa, 1994; Nunn, 1974; Bespalov and Trakhtengerts, 1974; Curtis, 1978). Being closely associated with the generation of spontaneous chorus emissions, there have been proposed two possibilities as stimulus to trigger a chorus. The first one is hiss which has so far been considered to be very random and turbulent, because the ground and satellite VLF/ELF measurements have indicated that chorus is frequently accompanied by a background of hiss (Burtis and Helliwell, 1976; Cornilleau-Wehrlin, 1978; Koons, 1981). Second candidate is power line harmonic radiation (PLHR) (Luette et al., 1979) which is monochromatic and coherent in nature. Based on the observations of hisstriggering chorus events by the SCATHA satellite, Koons (1981) has concluded that the structures or large-amplitude spectral components existing in the hiss band are able to phase-bunch the electrons, which excite the chorus emissions. However, at low latitudes this phenomena has not attracted the attention of many workers in this field, may be due to the lack of ground based observations. An understanding of the generation mechanism of these hiss-triggered chorus emissions observed at our low-latitude ground station Gulmarg would be most useful for inferring the properties of the high energy trapped electrons. With this aim, we have performed detailed signal analyses. In the present paper, we first present the analysis of the hiss-triggered chorus events observed at Gulmarg during the routine recording of whistlers. It is probably for the first time that the recording of such type of emissions has been reported from the 
low-latitude ground stations in India. From the analysis it is observed that each chorus elements starts from the hiss band and hence it is suggested that hiss has an important role in the generation of chorus in the outer magnetosphere. Further, a generation mechanism of hiss-triggered chorus emissions is proposed (Helliwell, 1967; Smirnova, 1984) based on the experimental results and various parameters associated with these emissions such as $L$-value of the source, source length etc. are estimated.

\section{Characteristics of the Dynamic Spectra and Re- lationship between Hiss and Chorus}

The present study is based on the VLF observations made at our low latitude ground station Gulmarg $(L=1.28)$ in India from January to July, 1986. These emissions recorded at Gulmarg are of good quality, and the number of emissions recorded during magnetic storm are large enough to be of statistical significance. VLF wide band signals (up to 10 $\mathrm{kHz}$ ) are received by a T-type of antenna (25 meter high and about 6 meter long horizontaly), which are suitably amplified by pre-and main-amplifiers, and recorded on magnetic tapes on the routine basis. We make the aural monitoring of the recorded data and when we find aurally any VLF signals, we display them as sonograms on VLF analyzers. VLF spectra of hiss-triggered chorus emissions in large numbers were detected during night time of March 8, 1986 at our field station Gulmarg during routine continuous recordind of VLF waves. No reports have so for appeared on the recording and morphological properties of such hiss-triggered chorus VLF emissions from any of the low latitude ground stations in India. The present finding is, therefore, believed to be the first such event reported from the low latitude ground stations in India. Attempts had been made to record such type of emissions at other Indian stations with little or no success. The reason of not detecting such type of emissions at other Indian stations may be non-occurrence of such type of emissions during the observation periods. The observation of hiss-triggered chorus emissions at the ground station Gulmarg is rare in the sence that most of the reported observations are mainly from the satellites (Hattori and Hayakawa, 1994).

A typical spectrogram of hiss-triggered chorus emissions observed at Gulmarg is shown in Fig. 1. The structureless signal below about $2.5 \mathrm{kHz}$ is called hiss, which is similar to band-limited white noise. Chorus emissions are those which have discrete rising tone structures. It is likely that each chorus element shown in the figure originates from the upper edge of the underlying hiss band and also that every riser is asymptotic to the hiss band at the begining, that is, every chorus has a very small initial $d f / d t$ value. The duration of each chorus elements is about $0.2 \mathrm{sec}$ and average $d f / d t$ is about $0.1554 \mathrm{kHz} / \mathrm{s}$. Also, the chorus occurrence rate is found to be closely correlated with the underlying intense hiss activity, that is, the chorus generation is enhanced when the hiss intensity becomes high.

These emissions were recorded at low-latitude ground station Gulmarg during the strong magnetic activity on March 7-9, 1986. Dst variations during the storm period is shown in Fig. 2, in which emissions events at Gulmarg are also marked. From the figure we find that the hiss-triggered chorus emissions on March 7, were not observed which may be due to

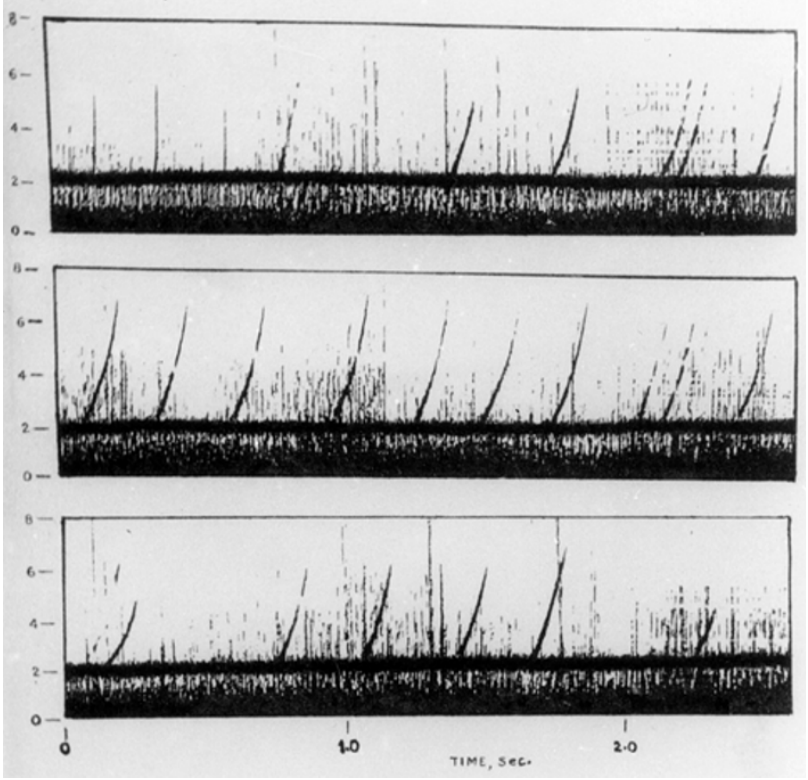

Fig. 1. A typical spectrogram of hiss-triggered chorus emissions observed at Gulmarg, 8 March, 1986.

the fact that the first substorm could not produce suitable conditions for the generation and registration of emissions at low-latitude stations. The emissions connected with the particle injection during the substorm could have been observed at higher latitudes. The powerful hiss-triggered chorus emissions were registered in the morning hours of March 8, after the substorm activity of March 7 . The highest magnetic ( $\Sigma K_{p}=34$ ) activity was observed on March 8, and also the most intensive events were registered in the morning of March 8 during the recovery phase of the substorm.

Hiss-triggered chorus events started at 0256 IST (Indian Standard Time)as shown in Fig. 1 (top). The short discrete chorus elements appeared in the frequency range of about $2.0-5.5 \mathrm{kHz}$ having a small $d f / d t$ value. In the course of time the upper boundary frequency $f_{\mathrm{UB}}$ began increasing and it reached $7.5 \mathrm{kHz}$ by 0350 IST (Fig. 1 (middle)). For the next two hours the upper boundary frequency $\left(f_{\mathrm{UB}}\right)$ remained approximately constant. From 0550 IST it started decreasing slowly and by 0653 IST (Fig. 1 (bottom)) it reached to 4.6 $\mathrm{kHz}$. The observed mean chorus element has the following parameters: $f_{\min }=2.4 \mathrm{kHz}, f_{\max }=6.5 \mathrm{kHz}$ and $d f / d t=$ $1554 \mathrm{~Hz} \mathrm{~s}^{-1}$. The standard deviation of $f_{\mathrm{UB}}$ is found to be about 0.74 .

\section{Generation Mechanism of the Hiss-Triggered Chorus Emissions}

The dynamic spectrum of the chorus events presented in the previous section suggests that these emissions are likely to have originated from the hiss band and they are also asymptotic to the hiss band. Recently, Hattori and Hayakawa (1994) analyzing the direction finding measurements have shown that the azimuthal angle values for both VLF and ELF hisstriggered chorus emissions are the same. Based on their measurements they have suggested that both phenomena come from the same source region. Further, they have discussed that a chorus event is triggered from a wavelet existing at 


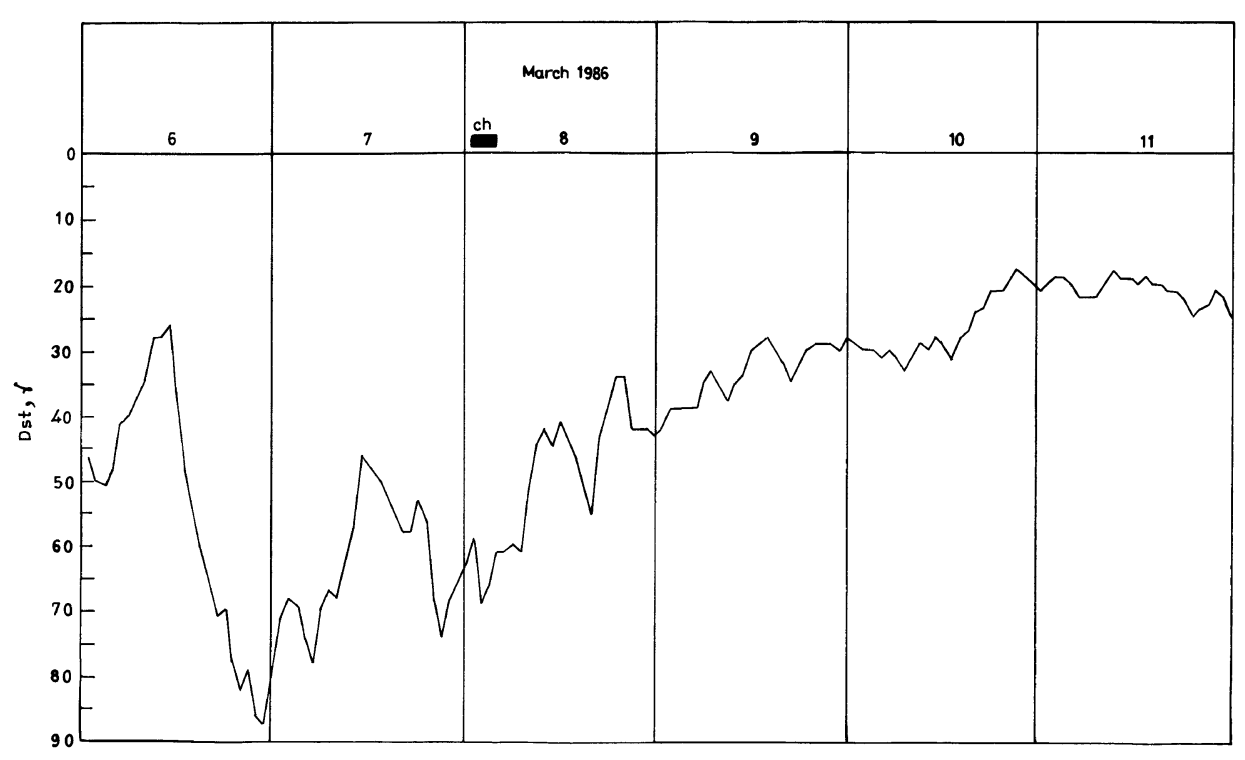

Fig. 2. Dst variation during the magnetic storm 6-11 March, 1986 chorus events are marked.

the upper edge of the hiss band through a coherent waveparticle interaction as was observed in the case of an active VLF injection experiment (Helliwell et al., 1986).

To explain the recorded spectrum at Gulmarg we propose to use the Helliwell's theory (Helliwell, 1967) of gyroresonance interaction between energetic streaming electrons and whistler mode waves travelling in opposite direction. This theory is able to explain most of the features of these emissions recorded at Gulmarg. The same mechanism has also been used by Hattori and Hayakawa (1994) to explain the hiss-triggered chorus emissions observed on board satellite GEOS 1. The above theory works in two steps: in the first case the wavelets located at the upper edge of the pre-existing hiss band having enough intensity and duration to interact resonantly with the energetic electrons phase bunch the interacting electrons. In the second step these phase-bunched electrons move away from the equator along the earth's magnetic field line and radiate coherently waves which satisfy the cyclotron resonance conditions and the dispersion conditions at the local latitude. The radiated waves propagate towards the equator and is detected at the observing stations. Hattori and Hayakawa (1994) have discussed the validity of the generation model through the reconstruction of chorus elements with the use of three-dimensional ray tracing computations.

The most frequent observations of chorus events by satellites near the geomagnetic equator (Tsurutani and Smith, 1974; Burtis and Helliwell, 1976) support the idea that the sources of chorus emissions most probably are localized near the equatorial region. The "magnetic equator" can be found well off the nominal position for dipole fields if the magnetosphere is compressed. Tsurutani and Smith (1977) showed that the "equator" can be quite elongated on the dayside where the solar wind pressure can form minimum B pockets. Smirnova et al. (1976) have argued that the source of chorus emissions is extremely localized in space and the appearance of chorus at the wide profile of observatories is the result of the propagational effect. The range of observed chorus frequencies is controlled by the equatorial electron gyrofrequencies (Burtis and Helliwell, 1969). The outer boundary of the plasmapause has the focussing property for the VLF chorus emissions. The maximum of chorus intensity is found to move towards lower latitudes with the increase in the geomagnetic activity which may be due to the motion of the propagating channels and source region towards the earth (Smirnova, 1984).

The above discussions clearly support the hypothesis that chorus emissions are triggered during wave particle interactions. In order to link the observed fine structure of the hiss-triggered chorus with the process of wave particle interaction in the corresponding magnetospheric region, we have followed the upper boundary frequency (UBF) method developed by Smirnova (1984) to find out the location of source for the chorus events. The upper boundary frequency of the ground-observed discrete VLF chorus is determined on the assumption of dipolar geomagnetic field configuration, by the half equatorial electron gyrofrequency in the generation region, irrespective of the latitude of the observation station. The $L$-value of the chorus source is then computed as

$$
L=\left(\frac{440}{f_{\mathrm{UB}}}\right)^{1 / 3} \text {. }
$$

Where $f_{\mathrm{UB}}$ is in $\mathrm{kHz}$. The upper boundary frequency $f_{\mathrm{UB}}$ of the observed chorus as shown in Fig. 1 is about $6.5 \mathrm{kHz}$ and hence the $L$-value of the source region for the reported event is found to be $L_{\text {Source }}=4.07$.

Helliwell (1967) has proposed a simple phasing criterion to estimate the length of the resonance region and other parameters of the discrete chorus emissions. According to him the expression for the source length $S$ along the dipolar geomagnetic field line is given by

$$
S=5.85 \times 10^{5} \frac{(1-\lambda)^{1 / 2}}{f_{\mathrm{N}}^{1 / 3} f_{\mathrm{HO}}^{2 / 9} \lambda^{1 / 6}} \mathrm{~km}
$$

where frequencies are expressed in $\mathrm{Hz}, \lambda=f / f_{\mathrm{HO}}, f$ is wave frequency, $f_{\mathrm{HO}}$ is electron gyrofrequency at the top of 
the path, and $f_{\mathrm{N}}$ is electron plasma frequency. At $L=4.07$, the electron density is $\sim 16$ electrons $\mathrm{cm}^{-3}$ (Carpenter, 1963; Angerami and Carpenter, 1966). The corresponding plasma frequency is $36 \mathrm{kHz}$. At the wave frequency of $2.4 \mathrm{kHz}$, the source length comes out to be about $2114 \mathrm{Km}$. The parallel velocity of resonantly interacting electron is given by

$$
V_{\|}=\frac{c\left(f_{\mathrm{H}}-f\right)^{3 / 2}}{\left(f_{\mathrm{N}} f^{1 / 2}\right)}
$$

where $c$ is velocity of light, $f_{\mathrm{H}}$ is electron cold plasma gyrofrequency. The corresponding parallel velocity of electron $V_{\|} \approx 1.6 \times 10^{5} \mathrm{~km} \mathrm{~s}^{-1}$, which is in good agreement with the results reported by Helliwell (1967) and Smirnova (1984). The phase bunching of electrons produces a spatial maximum in the transverse current density which in turn generates the observed radiation. The magnitude of the transverse current depends on the concentration of the charged particles, and on the magnitude and phasing of their transverse velocities. Assuming an isotropic distribution of pitch angles, the normalized current was found to peak at $30^{\circ}$ which we have taken as the characteristic pitch angle for the interaction. The corresponding characteristic value of the perpendicular velocity is given by $V_{\perp}=0.577 V_{\|}$(Helliwell, 1967). Using a simple phasing criterion method (Helliwell, 1967), the concentration of electrons in the transverse current stream is computed to be about $0.15 \mathrm{~m}^{-3}$. All these estimated parameters lie within the range of observations and are in good agreement with the results reported by Helliwell (1967) and Smirnova (1984).

\section{Conclusion}

A possible generation mechanism of hiss-triggered chorus emissions is presented based on experimental results of low-latitude ground station Gulmarg which has two steps: phase bunching of resonant electrons by a causative wavelet present in the hiss spectrum and continuous radiation by the phase bunched electrons through the cyclotron instability mechanism at different latitudes as the electrons travel away from the equator. This concept is based on the generation process of riser emissions in Helliwell's theory (Helliwell, 1967). Helliwell's theory as also the UBF method seem to be quite suitable for the interpretation of the discrete chorus fine structures observed at low-latitudes. The advantage of UBF method is that we can use the available data of any one station since the upper boundary frequency is expected to be the same at all the recording stations irrespective of their locations in latitude (Smirnova, 1984). To derive informations about the fine structures of the dynamic spectra and detailed informations about the generation mechanism, exhaustive work should be carried out to compare the various features of natural observations, active injection experiments and computer simulations.

In the present paper, an approach based on the natural observations is performed. Unfortunately, the results of fine structure analysis and direction finding for the VLF data reported in this paper is not available at our observing station Gulmarg in order to see the existence of the causative wavelets with significant duration of time at the foot of each chorus element. Not with standing these limitations, the present exercise of studying the generation mechanism of hiss-triggered chorus emissions observed at low-latitude is worthwhile. Hence, further study is required to explain the micro-processes of hiss-triggering chorus emissions observed at low-latitudes. Investigation of the micro-process to explain how a causative wavelet triggers a chorus emission at low-latitudes is a problem for the future.

Acknowledgments. The present work is supported by Department of Science and Technology, New Delhi under the SERC projects.

\section{References}

Angerami, J. J. and D. L. Carpenter, Whistler studies of the plasmapause in the magnetosphere, 2 , electron density and total tube content near the knee in magnetospheric ionization, J. Geophys. Res., 71, 711-725, 1966. Bespalov, P. A. and V. V. Trakhtengerts, Non stationary particles distribution in the magnetosphere and generation of periodic emissions in the VLF and SPP range, Geomagn. Aeronom., 14, 266-271, 1974.

Burtis, W. J. and R. A. Helliwell, Banded chorus-A new type of VLF radiation observed in the magnetosphere by OGO-1 and OGO-3, J. Geophys. Res., 74, 3002-3010, 1969.

Burtis, W. J. and R. A. Helliwell, Magnetospheric chorus; Occurrence patterns and normalized frequency, Planet. Space Sci., 24, 1007-1024, 1976.

Burton, R. K. and R. E. Holtzer, The origin and propagation of chorus in the outer magnetosphere, J. Geophys. Res., 79, 1014-1023, 1974.

Carpenter, D. L., Whistler evidence of a knee in the magnetospheric ionization density profile, J. Geophys. Res., 68, 1675-1682, 1963.

Cornilleau-Wehrlin, N., R. Gendrin, F. Lefeuvre, M. Parrot, R. Grard, and D. Jones, VLF waves observed onboard GEOS-1, Space Sci. Rev., 22, 371-382, 1978.

Curtis, S. A., A theory for chorus generation by energetic electrons during substorms, J. Geophys. Res., 83, 3841-3848, 1978.

Goldstein, B. E. and B. T. Tsurutani, Wave normal direction of chorus near equatorial source region, J. Geophys. Res., 89, 2789-2805, 1984.

Hattori, K. and M. Hayakawa, Consideration of dynamic spectra and direction finding results of hiss-triggered chorus emissions, Proc. NIPR Symp Upper Atmos. Phys., 7, 40-52, 1994.

Hayakawa, M., Y. Yamanaka, M. Parrot, and F. Lefeuvre, The wave normals of magnetospheric chorus emissions observed onboard GEOS-2, $J$. Geophys. Res., 89, 2811-2821, 1984.

Hayakawa, M., K. Hattori, S. Shimakura, M. Parrot, and F. Lefeuvre, Direction finding of chorus emissions in the outer magnetosphere and their generation and propagation, Planet. Space Sci., 38, 135-141, 1990.

Helliwell, R. A., Whistlers and Related Ionospheric Phenomena, 349 pp., Stanford Univ. Press, Stanford, U.S.A, 1965

Helliwell, R. A., A theory of discrete VLF emissions from the magnetosphere, J. Geophys. Res., 72, 4273-4790, 1967.

Helliwell, R. A., D. L. Carpenter, U. S. Inan, and J. P. Katsufrakis, Generation of band-limited VLF noise using the Siple transmitter: A model for magnetospheric hiss, J. Geophys. Res., 91, 4381-4392, 1986.

Koons, H. C., The role of hiss in magnetospheric chorus emissions, J. Geophys. Res., 86, 6745-6754, 1981.

Luette, J. P., C. G. Park, and R. A. Helliwell, The control of magnetosphere by power line radiation, J. Geophys. Res., 84, 2657-2660, 1979.

Nunn, D., A theoretical investigation of banded chorus, J. Plasma Phys., 11, 189-212, 1974.

Sazhin, S. S., Natural Radio Emissions in the Earth's Magnetosphere, Nauka, Moskow, 1982.

Sazhin, S. S. and M. Hayakawa, Magnetospheric chorus emissions: A review, Planet. Space Sci., 40, 681-697, 1992.

Smirnova, N. A., Yu. P. Novikov, N. G. Kleimenova, and E. E. Titova, Some spectral peculiarities of VLF emissions registered on the earth surface near the plasmapause projection, J. Atmos. Terr. Phys., 38, 1217, 1976.

Smirnova, N. A., Fine structure of the ground observed VLF chorus as an indicator of the wave particle interaction process in the magnetosphere, Planet. Space Sci., 32, 425-438, 1984.

Tsurutani, B. T. and E. J. Smith, Postmidnight chorus: A substorm phenomenon, J. Geophys. Res., 79, 118-127, 1974.

Tsurutani, B. T. and E. J. Smith, Two types of magnetospheric ELF chorus and their substorm dependences, J. Geophys. Res., 82, 51120, 1977.

R. Singh, R. P. Patel, R. P. Singh (e-mail: rampal@banaras.ernet.in), and Lalmani 